\title{
TARMED-Info
}

\author{
Bericht Nr. 17
}

Tarifdienst FMH

\section{TARMED-Fragen Payline}

Die Einführung von TARMED führt erwartungsgemäss zu vielen Anfragen beim Tarifdienst. Um die Fragen effizient beantworten zu können, werden ab Montag, 19. Januar 2004, gemäss Beschluss der Präsidentenkonferenz vom 4. Dezember 2003 folgende Paylines aufgeschaltet:

\section{Vorgaben zum Gebrauch der beiden Telefonhotlines}

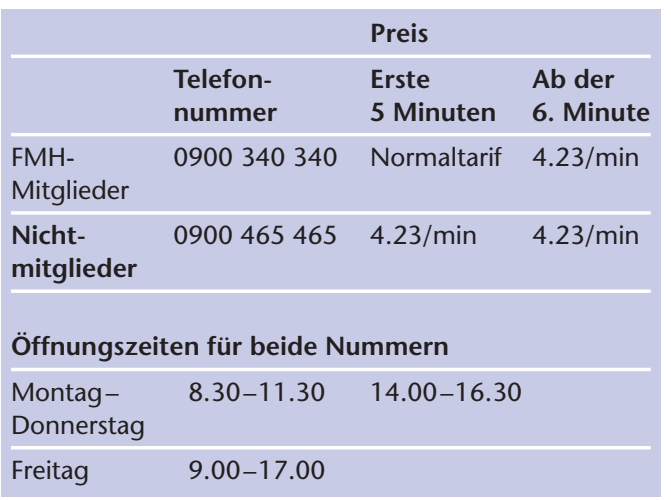

\section{Es sind dabei die folgenden Regeln} zu beachten

\section{Sprache}

a) Deutsch oder Französisch. Die gewünschte Sprache wird vor Beginn des gebührenpflichtigen Gesprächs erfragt.

b) Französische Anfragen werden generell nur mittwochs und freitags beantwortet (Ausnahmen davon werden vor Beginn des Gesprächs mitgeteilt).

\section{Linienzahl}

Es ist Montag bis Freitag jeweils eine Person mit der Telefonbeantwortung beschäftigt. Das heisst: Wird ein Anruf auf die eine oder andere Payline beantwortet, sind jeweils beide Rufnummern besetzt.

\section{E-Mail, Briefe und Faxanfragen}

Ist die anfragende Person tatsächlich FMH-Mitglied, werden einfache Fragen kurz beantwortet. In allen komplizierteren Fällen und bei Anfragen von Nichtmitgliedern wird auf die Payline verwiesen.

\section{Andere Telefonlinien der FMH}

Anfragen betreffend TARMED auf die Telefonnummer des FMH-Tarifdienstes bzw. der FMHConsulting oder des Generalsekretariates werden ebenfalls auf die Paylines verwiesen.

Es ist wichtig, dass Sie Ihre Anfragen so vorbereiten, dass inkl. der Antwort nicht mehr als 5 Minuten benötigt werden, damit FMH-Mitglieder nicht den erhöhten Tarif zahlen müssen und die Leitungen nicht dauernd besetzt sind. Weiter behalten wir uns vor, mit 1 bis 2 Kontrollfragen zu prüfen, ob es sich bei der anrufenden Person tatsächlich um ein FMH-Mitglied handelt.

\section{FAQ}

Wie sind Blutentnahmen kapillär und venös für den Arzt in der Praxis verrechenbar? Gemäss neuer Version der eidgenössischen Analysenliste können im Praxislabor keine Leistungen des Kapitels 4.1 Allgemeine Leistungen mehr verrechnet werden.

Diese Lösung ist für die Ärzteschaft unbefriedigend. Es sind aktuell Verhandlungen am laufen, um diesen Umstand zu korrigieren.

\section{Gibt es einen Mac-TARMED-Browser?}

Die FMH stellt jeweils als Dienstleistung an die Mitglieder eine aktuelle «Ansichtsversion» des TARMED-Browsers für Mac-User in deutsch, französisch und italienisch zur Verfügung. Der Browser wird von der ZMT (Zentralstelle für Medizinaltarife) jeweils nur in der Windowsversion zur Verfügung gestellt.

Nachteil für MAC-User: «Rollbalken» können in den Mac-Versionen nicht generiert werden. Es kann aber mit den «Pfeiltasten» geblättert werden. Ebenfalls fehlt die Suchfunktion auf den Mac-Versionen. Es ist mit normalem Aufwand nicht möglich, einen Browser in Form der Windows-Versionen für Mac zu erstellen. 
Sind die elektronischen Rechnungsversionen und oder Handabrechnungsformulare bereits bekannt?

Im TARMED-UVG-Bereich sind die Formulare schon seit längerem abrufbar, u. a. auf der Internetseite www.fmh.ch/Tarife/Tarmed. Im KVGBereich wurde das Handabrechnungsformular vor wenigen Tagen ebenfalls aufgeschaltet. Das elektronische Formular im KVG-Bereich ist zurzeit noch nicht ausgearbeitet, es sollte aber in den nächsten Tagen soweit sein und wird dann ebenfalls auf der Homepage der FMH abrufbar sein.

\section{TARMED-Info}

\section{Bulletin $\mathrm{N}^{\circ} 17$}

Service tarifaire FMH

\section{Questions au sujet de I'utilisation du TARMED - Payline}

L'introduction du TARMED (LAMal) a pour conséquence une augmentation substantielle du nombre de questions posées au Service tarifaire de la FMH. Comme approuvé lors de la Conférence des Présidents du 4 décembre 2003 et afin de pouvoir faire face au mieux à l'afflux de questions, une ligne téléphonique payante sera installée dès le lundi, 19 janvier 2004.

\section{Les conditions d'utilisation de ces numéros de téléphone payants sont les suivantes}

\begin{tabular}{|c|c|c|c|}
\hline \multicolumn{4}{|c|}{ Prix } \\
\hline & $\begin{array}{l}N^{\circ} \text { de télé- } \\
\text { phone }\end{array}$ & $\begin{array}{l}\text { Premières } \\
5 \text { minutes }\end{array}$ & $\begin{array}{l}\text { Dès la } \\
6^{\mathrm{e}} \text { minute }\end{array}$ \\
\hline $\begin{array}{l}\text { Membres } \\
\text { FMH }\end{array}$ & 0900340340 & Tarif normal & 4.23 par min \\
\hline $\begin{array}{l}\text { Non- } \\
\text { membres }\end{array}$ & 0900465465 & 4.23 par min & 4.23 par min \\
\hline \multicolumn{4}{|c|}{ Horaire (pour les deux numéros) } \\
\hline $\begin{array}{l}\text { Lundi } \\
\text { à jeudi }\end{array}$ & $8 h 30-11 h 30$ & $14 \mathrm{~h} 00-16 \mathrm{~h} 30$ & \\
\hline Vendredi & $9 \mathrm{~h} 00-17 \mathrm{~h} 00$ & & \\
\hline
\end{tabular}

\section{Quelques règles d'utilisation sont à considérer}

\section{Langue}

a) Français ou allemand, choix guidé par menu (avant le début de la communication surtaxée).

b) En principe, il ne sera répondu en français que les mercredis et vendredis (information donnée avant le début de la communication surtaxée).

\section{Nombre de lignes}

Les deux numéros payants aboutissent sur une seule ligne. Cela signifie que dès que cette ligne est occupée par un appelant, les deux numéros donnent pour un nouvel appelant la tonalité «occupé».

\section{E-mail, lettre ou fax}

Pour autant que l'expéditeur soit effectivement un membre, seules les questions simples et ne nécessitant pas de grandes explications seront considérées. Un triage dans ce sens sera fait et la réponse pourra dès lors demander à l'expéditeur de prendre contact sur la ligne payante. 
Autres lignes téléphoniques de la FMH

Les appels concernant des questions au sujet du TARMED sur d'autres numéros de la FMH, tel que par exemple sur le numéro de téléphone Secrétariat général renverront systématiquement sur les lignes payantes.

Nous conseillons donc vivement aux membres de la FMH de bien préparer leurs questions afin que la conversation téléphonique ne dépasse pas 5 minutes. De plus, cela permet de libérer rapidement la ligne pour d'autres appelants. Nous leur conseillons également à se préparer à répondre à 1 ou 2 questions qui permettent de certifier leur qualité de membre.

\section{FAQ}

Les prise de sang (capillaire ou veineux) pour analyse de laboratoire sont-elles facturables? Selon la Liste des analyses 2004 édictée par le DFI, il n'est plus pour un cabinet médical possible de facturer les positions générales du chapitre 4.1.

Cette situation est inacceptable pour les médecins et des négociations sont en cours pour remédier à cette situation.
Existe-t-il un navigateur TARMED pour Mac?

La FMH met à disposition des utilisateurs d'ordinateurs Mac une plate-forme qui permet de visualiser le TARMED. Cette version est préparée sur la base de la version Windows du navigateur TARMED qui est mise à disposition de la FMH par la Commission des Tarifs Médicaux.

Cette version Mac souffre d'une faiblesse. En effet la navigation par les «ascenseurs» (scroll bars) n'est pas possible et ce sont les touches «flèches» (haut/bas) du clavier qu'il faut dès lors utiliser pour naviguer dans le tarif. Il n'est malheureusement pas possible de remédier simplement et sans d'excessifs investissements à ce problème qui relève de la situation compétitive entre deux systèmes informatiques.

\section{Les formules de facturations électroniques} existe-t-elles?

Les formules à utiliser pour les tarifs LAA/LAI/ LAM existent de longue date. Les formules pour utilisation en LAMal pour la facturation «par main» existent également. Le formulaire pour la facturation électronique soit fermé par clef dès qu'il est élaboré. Il faut à ce titre consulter le site Internet de la FMH (www.fmh.ch) ou ces documents peuvent être téléchargés. 